\author{
M. Steiner - B. Steiner - A. Rolfs - M. Wangnick - C. Burstein • \\ M. Freund $\cdot$ P. Schuff-Werner
}

\title{
Antithrombin gene mutation 5356-5364*deICTT with type I deficiency and early-onset thrombophilia and a brief review of the antithrombin $\alpha$-helix D molecular pathology
}

Published online: 13 April 2005

(C) Springer-Verlag 2005

The online version of the original article can be found at http://dx. doi.org/10.1007/s00277-004-0906-3

\section{Ann Hematol (2004) 84:56-58}

In the original publication of this article, Tables 1 and 2 were unfortunately omitted. We apologise for this mistake. The correct versions of Tables 1 and 2 are given below.

Table 1 Antithrombin $\alpha$-helix D amino acid residues with their major structural and functional impact. Mutation entries apply to human case studies only, whereas in vitro studies have generally not been considered

\begin{tabular}{|c|c|c|c|}
\hline Residue & Structural/functional impact & Mutation & References \\
\hline Phe $121-123$ & $\begin{array}{l}\text { Helix packing, heparin } \\
\text { binding, protein folding }\end{array}$ & delPhe, type I deficiency, misfolding & $\begin{array}{l}{[5,16,24} \\
\text { this report }]\end{array}$ \\
\hline Lys 125 & Heparin binding & & \\
\hline Leu 126, Asn 127 & $\begin{array}{l}\text { Helix packing, protein } \\
\text { folding }\end{array}$ & Leu126Pro, type I deficiency, misfolding & {$[25]$} \\
\hline Cys 128 & $\begin{array}{l}\text { Disulfide bond formation } \\
\text { with Cys } 8 \\
\text { conformation of the } \\
\text { heparin-binding site }\end{array}$ & $\begin{array}{l}\text { Cys128Tyr, type I deficiency, misfolding, formation of } \\
\text { high molecular weight } \\
\text { AT species, impaired heparin-binding affinity }\end{array}$ & [7] \\
\hline Leu 130, Tyr 131 & $\begin{array}{l}\text { Helix packing, protein } \\
\text { folding }\end{array}$ & & \\
\hline Arg 132, Lys 133 & $\begin{array}{l}\text { Interaction with shutter } \\
\text { region, heparin binding }\end{array}$ & Arg132stop, type I deficiency & {$[25]$} \\
\hline Ala 134 & $\begin{array}{l}\text { Linker between } \alpha \text {-helix } \\
\mathrm{D} \text { and } \beta \text {-sheet } \mathrm{A} \\
\text { heparin binding }\end{array}$ & & \\
\hline Asn 135 & $\begin{array}{l}\text { Glycosylation site } \\
\text { minor fraction unglycosylated }(\beta-\mathrm{AT})\end{array}$ & $\begin{array}{l}\text { Asn135Thr, borderline AT deficiency, asymptomatic, } \\
\text { glycosylation block, increased } \beta \text {-AT with high heparin affinity }\end{array}$ & {$[3]$} \\
\hline
\end{tabular}

M. Steiner $(\bowtie) \cdot$ M. Wangnick $\cdot$ C. Burstein P. Schuff-Werner

Institute of Clinical Chemistry and Pathobiochemistry,

University of Rostock,

Ernst-Heydemann-Strasse 6,

18057 Rostock, Germany

e-mail: michael.steiner@med.uni-rostock.de

Tel.: +49-381-4947591

Fax: +49-381-4947672
B. Steiner · M. Freund

Division of Haematology/Oncology,

Department of Internal Medicine,

University of Rostock,

Rostock, Germany

A. Rolfs

Department of Neurology,

University of Rostock,

Rostock, Germany 
Table 2 Antithrombin (AT) gene mutation 5356-5364*delCTT leading to a deletion of a phenylalanine residue at codons $121-123$. Summary of reported cases. DVT deep vein thrombosis, $P E$ pulmonary embolism

\begin{tabular}{lllll}
\hline Patient & AT activity (\%) & AT antigen (\%) & Comments & Reference \\
\hline 1 & 62 & 73 & & {$[5]$} \\
2 & 47 & 48 & Thrombophlebitis & {$[16]$} \\
3 & 55 & 41 & DVT/PE & {$[24]$} \\
4 & 38 & 43 & Multiple DVT & This report \\
\hline
\end{tabular}

